Max-Planck-Institut für demografische Forschung

Max Planck Institute for Demographic Research

Konrad-Zuse-Strasse 1 - D-18057 Rostock · GERMANY

Tel +49 (0) 3812081 - 0; Fax +49 (0) 3812081 - 202;

http://www.demogr.mpg.de

MPIDR WORKING PAPER WP 2010-018

FEBRUARY 2010

\title{
A discrete-time model of metabolic adaptation to recurring diet changes of Medfly females
}

Arseniy S. Karkach (eburg@inm.ras.ru)

Alexey A. Romanyukha (arseny@mail.ru)

Anatoli I. Yashin (aiy@duke.edu)

This working paper has been approved for release by: James W. Vaupel (jwv@demogr.mpg.de), Head of the Laboratory of Survival and Longevity.

(C) Copyright is held by the authors.

Working papers of the Max Planck Institute for Demographic Research receive only limited review. Views or opinions expressed in working papers are attributable to the authors and do not necessarily reflect those of the Institute. 


\title{
A discrete-time model of metabolic adaptation to re- curring diet changes of Medfly females
}

\author{
MPIDR working paper
}

August 2009

Arseniy S. Karkach ${ }^{1}$, Alexey A. Romanyukha ${ }^{1}$, Anatoli I. Yashin ${ }^{2}$

\begin{abstract}
${ }^{1}$ Institute of Numerical Mathematics, Russian Academy of Sciences, Gubkina 8, 119333, Moscow, Russia. Phone: +7 (495) 938 3765, Email: eburg@,inm.ras.ru; +7 (495) 938 3990; arseny@mail.ru

${ }^{2}$ Duke University, Center for Demographic Studies, 2117 Campus Drive, Box 90408, Durham, NC 27708-0408, USA
\end{abstract}

\section{Introduction}

Carey et al (2002) performed an experiment in which they studied the response of Mediterranean fruitfly (C. capitata) females in terms of individual longevity and fertility to pulsed changes in diet quality. Our analysis of the data (Romanyukha et al. 2009) discovered a pronounced trade-off between the average fertility and longevity of Medfly fe males on different diets among the longlived and most fertile part of the population. In order to analyze and describe the observed phenomenon we constructed the mathematical model of metabolic adaptation.

The model is based on the knowledge about physiological mechanisms underlying the metabolic regulation in insects (Simmons and Bradley, 1997; Harshman and Zera, 2007). The experimental data provides reproductive life histories for groups of individuals. The model is constructed on two levels. The individual level describes the life history of a single fly female. It models the change of the organism state with time in response to parameters of the environment. The outputs of individual model are individual life span and reproductive schedule. The population-level model describes a population of such individuals which differ randomly in values of certain parameters. The population level model allows studying population demography - i.e. survival, distribution of reproductive effort, fitness, etc.

The change of state in the individual-level model occurs as a result of metabolic processes occurring in the body. Two components of the fly's metabolism are considered the most important and are modeled: reproductive processes and somatic maintenance. They require different resources and the dynamics of those is modeled. The model describes changes in three body resources. Two of them are metabolic and irreplaceable, one used for maintenance and the other for reproduction. The third resource is replaceable from food. The parameters of the individual and population model were estimated from literature and by fitting to the data. 


\section{The data}

We analyzed the data of study of Carey et al. (2002) which is described here only briefly. A more detailed analysis is given in (Romanyukha, Karkach, 2009). Pairs of Mediterranean fruit fly females and males were subjected to different dietary regimes composed of two diets: the complete diet containing yeast hydrolyzate and sugar (diet $p$ ) and a sugar-only diet (diet $s$ ). Female life span and the number of eggs laid daily were studied in several cohorts, which received food in cycles of one day diet $p$ and 1,3,5,10, or 20 days diet $s$. One control cohort received diet $s$ full time and one received diet $p$ full time.

The average life spans in cohorts did not show high response to changes in the diet. In contrast the total fertility averaged by group experienced substantial change in response to diet changes (see Fig. 1, circles). Although within each cohort there was high variability in individual fertility and longevity, there was no evidence of a reproduction-longevity trade-off.

To deeper investigate variability of life history traits in response to diet changes we subdivided the flies in each cohort into three groups by life span: $25 \%$ flies with the highest life span, $25 \%$ with the lowest and 50\% with intermediate life span, and calculated average life spans and average total fertility in groups. This revealed much higher variability in both life history traits among the long lived flies, than among the short-lived ones. The life spans in groups of longlived flies has the highest variability. The same groups demonstrated higher variability or fertility ${ }^{1}$.

We denote the cohorts which received dietary regime composed of one day protein $(p)$ and $\mathbf{n}$ days sugar $(s)$ with a number such as 1, 3, 5, 10, and 20 which means 1-to-1, 1-to-3, 1-to-5 etc. type of diet. The controls are denoted as $\mathbf{p}$ and $\mathbf{s}$. Fig. 1 displays average lifespan and fertility for the entire cohorts $1,3,5,10,20, p$, and $\mathbf{s}$ and groups of short- and long-lived flies. One can see a negative correlation between average fertility and life span in long-lived flies; it is approximated by the straight line. Relation between the traits depends on the amount of diet $p$ in the ration: more $p$-days lead to higher fertility and shorter life span. Cohort $\mathbf{s}$ is an outlier because the females can not realize their reproductive potential on sugar diet. For flies in short-lived groups (squares) all diets result in closer and relatively small values of average lifespan and reproduction. A similar connection between fertility and life span exists in groups of flies selected by fertility (See Fig. 1, inset).

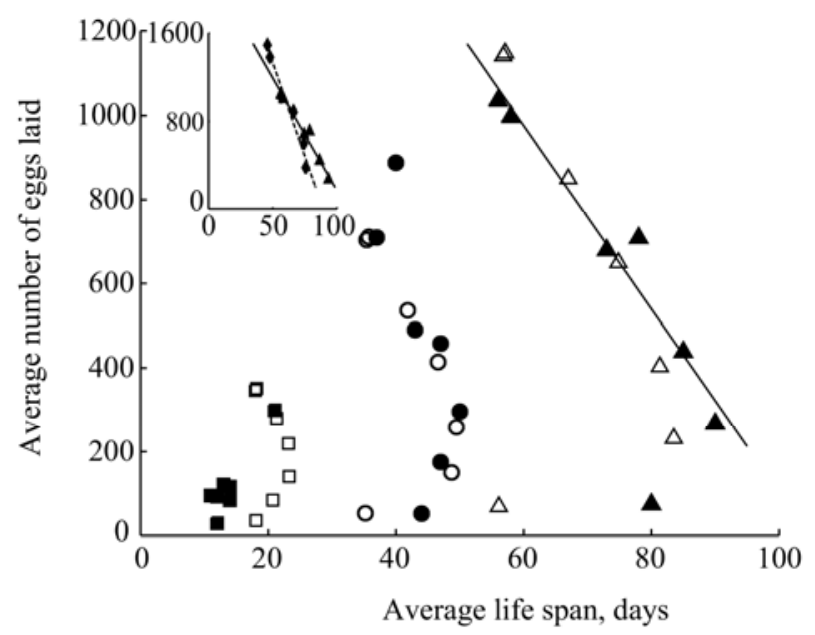

Fig. 1. Average life span and average fertility (number of laid eggs) of flies on different diets in groups of $25 \%$ short-lived (squares), 25\% long-lived (triangles) and all flies (circles) in experiment (filled) and model (open symbols). The regression line is fitted to the average points

\footnotetext{
${ }^{1}$ Selecting other proportion of flies as "long-lived" or "most reproductive" give similar results and also demonstrate a trade-off although with a different slope of the regression line.
} 
in the long-lived groups. The inset shows averages and regression lines for the $25 \%$ long-lived (triangles, line) and 25\% most fertile (diamonds, broken line).

\section{The model of individual}

The model describes survival and daily egg production by an individual female fruit fly. It is based on modeling of state change in response to environmental conditions. The modeled "control system" of an individual fly is a simplified representation of molecular and genetic mechanisms of the body control. Our analysis of the data of the cyclic diet experiments (Carey et al., 2002) suggests that the adaptation of $C$. capitata females to recurring diet changes may be described by three quantitative and one qualitative state characteristic which we call resources. Two of them characterize metabolic processes of somatic maintenance and reproduction. Assume a fly has metabolic resource $\mathrm{R}$ which is composed of two components: $R_{m}$ the resource used for maintenance and regeneration, and $R_{f}$ the one used for reproduction. Assume that at eclosion a fly starts life with a total amount of resources $R_{0}$ which is then allocated between two components: $R_{f}=c R_{0}$ and $R_{m}=(1-c) R_{0}$ where $c$ is the allocation coefficient (Romanyukha et al. 2004). Resource $R_{f}$ decreases proportionally to the number of eggs laid and $R_{m}$ proportionally to time lived. When $R_{f}=0$ the egg laying stops, when $R_{m}=0$ the fly dies.

Such introduction of non-replaceable resources assumes that these resources are essential for the corresponding function, they are depleted proportionally to the function performed and that they can not be replaced. Possible candidates for such kind of resource are the mitochondria. They are located in tissues and supply energy in the form of ATP to the processes occurring in these tissues and hence are essential. Mitochondria may get damaged in the process of energy generation due to generation of oxidative radicals and their power-generating capacity degrades. The oxidative damage may be assumed to be proportional to the generated energy which is closely proportional to the performed function.

Description of reproduction requires another resource - an essential nutrient required for egg formation which is received with food on diet $\mathrm{p}$. This nutrient may be a component of yeast hydrolyzate such as proteins, amino acids or sterols which are precursors for the hormone synthesis. We denote its amount $\Lambda$. When $\Lambda=0$ reproduction stops even if $R_{f}>0$.

The data of experiments with C. capitata suggest that there are two metabolic modes in which the flies can be (Carey et al. 1998): a waiting mode in which both mortality and reproduction are low and a reproductive mode in which mortality is very low at the onset of egg laying but accelerates as eggs are laid. Medflies stay in waiting mode when they are fed only sugar. When fed protein, a scarce resource in the wild, medflies switch to reproductive mode. In the model we define a mode $\mathrm{S}$ which can take values $\mathbf{m}$ - "maintenance" and $\mathbf{f}$ - "reproduction".

Hence our model of the organism defines state by three quantitative and one qualitative variables (Table 2): $R_{f}$ - metabolic resource allocated to reproduction, $R_{m}$ - metabolic resource allocated to maintenance (both are non-replaceable resources which can be reallocated), $\Lambda$ essential resource replaceable from diet, and the binary variable "metabolic mode", S.

Table 1. Individual state variables

\begin{tabular}{l|l}
\hline Variable & Comments \\
\hline$R_{f}$ & Amount of metabolic resource allocated to reproduction \\
$R_{m}$ & Amount of metabolic resource allocated to maintenance \\
$\Lambda$ & Amount of dietary resource in the body \\
$S$ & Current metabolic mode of the fly $(\mathbf{m} / \mathbf{f})$ \\
\hline
\end{tabular}




\section{Environment}

The environment is defined by the type of diet, $D(x)$, which is offered to flies on a particular day of life. It can take one of two possible values: $\mathbf{s}$ for sugar diet and $\mathbf{p}$ for complete adult diet. Hence $D(x)$ is a piece-wise function constant throughout the day.

\section{Life history events}

The individual level model describes several events which are listed in Table 2.

Table 2. Events described by the individual model

\begin{tabular}{|c|c|}
\hline Event & Comments \\
\hline $\begin{array}{l}1 \text { - initial allocation of metabolic } \\
\text { resource }\end{array}$ & $\begin{array}{l}\text { In-born metabolic resource } R \text { is allocated between } R_{m} \\
\text { and } R_{f} \text { according to intrinsic (hereditary and epigenetic) } \\
\text { proportion }\end{array}$ \\
\hline $\begin{array}{l}2 \text { - reallocation of metabolic re- } \\
\text { source and change of metabolic } \\
\text { mode }\end{array}$ & $\begin{array}{l}\text { Adaptation to certain conditions such as a strong change } \\
\text { in environment. Reallocation of } R_{m} \text { and } R_{f} \text { may be ac- } \\
\text { companied by a change between "maintenance" and "re- } \\
\text { production" modes }\end{array}$ \\
\hline 3 - depletion of dietary resource $\Lambda$ & Stops reproduction \\
\hline $\begin{array}{l}4-\text { depletion of metabolic resource } \\
R_{f} \text { allocated to reproduction }\end{array}$ & Stops reproduction \\
\hline $\begin{array}{l}5-\text { depletion of metabolic resource } \\
R_{m} \text { allocated to maintenance }\end{array}$ & Causes fly death \\
\hline $\begin{array}{l}6 \text { - penalty for reallocation attempt } \\
7 \text { - change of metabolic mode, } S\end{array}$ & $\begin{array}{l}\text { Happens when the adaptation to changes in environment } \\
\text { conditions is attempted at low level of metabolic resource } \\
\text { The flies switch between two metabolic modes: "mainte- } \\
\text { nance" or "reproduction". They start life in random mode. } \\
\text { The switch depends on the level of dietary resource in the } \\
\text { body }\end{array}$ \\
\hline
\end{tabular}

\section{Model algorithm and parameters}

The model is constructed in discrete time with time step 1 day and is described by an algorithm shown in Fig. 2. The model parameters and variables are given in Table 3.

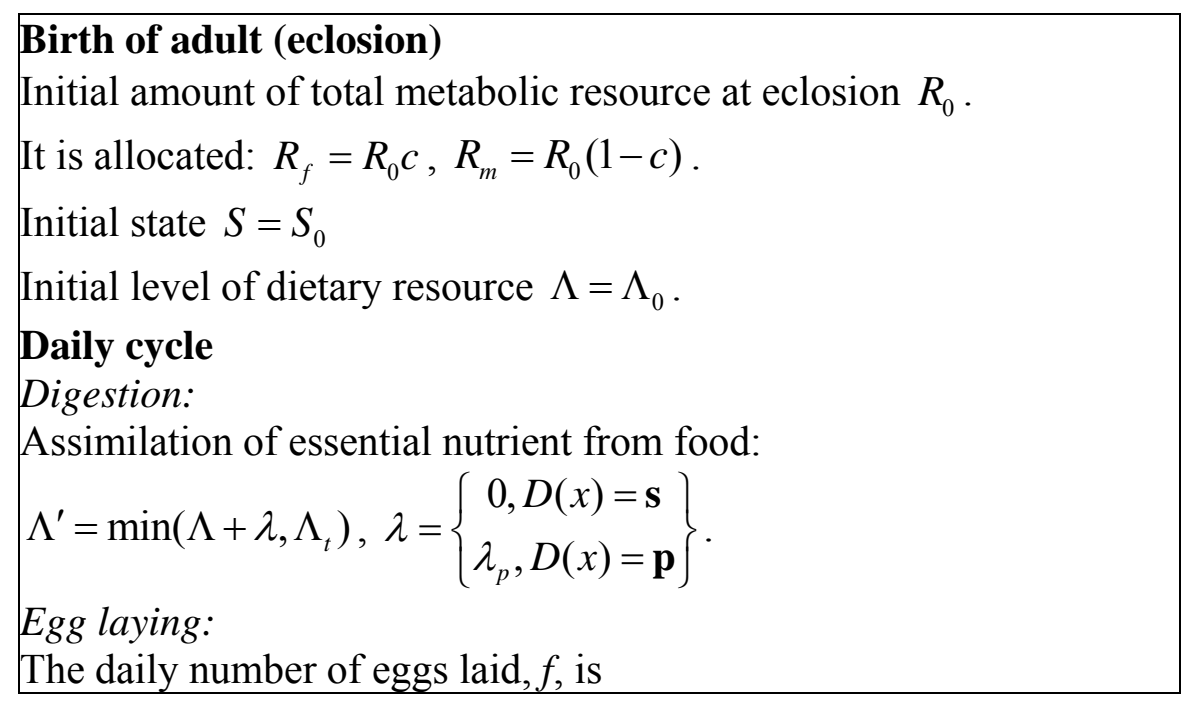




$f=\left\{\begin{array}{l}\quad 0, x<3 \\ \min \left(f_{\max }, R_{f} / \alpha_{f}, \Lambda / \rho\right), x \geq 3\end{array}\right.$
(reproduction does not start before day 3)
Resource usage:
$R_{f}^{\prime}=R_{f}-\alpha_{f} f ;$
$R_{m}^{\prime}=R_{m}-\alpha_{m} ;$
$\Lambda^{\prime}=\Lambda-\rho f$.
State change and resource reallocation:
If $S=\mathbf{m}$ and $\Lambda \geq \Lambda_{m f}$ then a state change occurs and $S^{\prime}=\mathbf{f}$.
State change is accompanied by a resource reallocation:
$R_{f}^{\prime}=c\left(R_{f}+R_{m}\right) ;$
$R_{m}^{\prime}=(1-c)\left(R_{f}+R_{m}\right)$
If $S=\mathbf{f}$ and $\Lambda<\Lambda_{m f}$ then the state changes $S^{\prime}=\mathbf{m}$ but resource is
not reallocated. Resource is reallocated only when switching from
mode $\mathbf{m}$ to $\mathbf{f}$.
Death
Occurs when the maintenance resource is depleted:
$R_{m}=0$.

Fig. 2. Algorithm of the model. Here $x$ is the fly's age, it is suppressed for brevity. The dash sign (') denotes the variable value at the next time step.

Table 3. Variables, parameters and characteristics of the solution for the model of individual life history adapting to repeating diet changes

\begin{tabular}{ccl}
\hline Notation & Value & Description \\
\hline $\begin{array}{cc}\text { Environment } \\
\text { Individual variables }\end{array}$ & Diet at age $x$, takes one of two values: $\mathbf{p}$ and $\mathbf{s}$ \\
$x$ & day & Age from eclosion \\
$S$ & $\mathbf{m} / \mathbf{f}$ & Functional state, takes one of two values \\
$R_{f}$ & $\mathrm{MU}$ & Level of reproduction resource \\
$R_{m}$ & $\mathrm{MU}$ & Level of maintenance resource \\
$\Lambda$ & $\mathrm{U}$ & Level of essential resource \\
Individual parameters & \\
$R_{0}$ & $\mathrm{MU}$ & Initial level of metabolic resource \\
$c$ & 0.45 & Parameter of metabolic resource (re)allocation \\
$S_{0}$ & $\mathbf{m} / \mathbf{f}$ & Initial state (mode) of the organism \\
$\alpha_{f}$ & 0.04 & Production cost of one egg (metabolic resource) \\
$\alpha_{m}$ & MU/day & Cost of one life day (metabolic resource) \\
$\rho$ & 1 & Production cost of one egg (dietary resource) \\
$\Lambda_{0}$ & $\mathrm{U}$ & Initial level of dietary resource \\
$\Lambda_{t}$ & 70 & Maximum level of dietary resource \\
$\lambda$ & $50 \mathrm{U} /$ day & Rate of dietary resource uptake on diet $\mathbf{p}$ \\
$\Lambda_{m f}$ & 10 & Threshold value for mode switch
\end{tabular}




\begin{tabular}{ccc}
$f_{\max }$ & 25 day $^{-1}$ & Maximum daily reproduction absent other limitations \\
Individual life & history characteristics \\
$f$ & day $^{-1}$ & Egg production rate \\
$F$ & & Number of eggs laid during lifespan \\
$X$ & day & Lifespan \\
Parameters & of the population model \\
$R_{0}$ & $\Gamma\left(\bar{R}_{0}=63, \sigma_{R}=28\right)$ \\
$\Lambda_{0}$ & $\Gamma\left(\bar{\Lambda}_{0}=55, \sigma_{\Lambda_{0}}=16\right)$ \\
$S_{0}$ & Binomial: $\{\mathbf{m}, \mathrm{p}=0.5 ; \mathbf{f}, \mathrm{p}=0.5\}$ \\
\hline
\end{tabular}

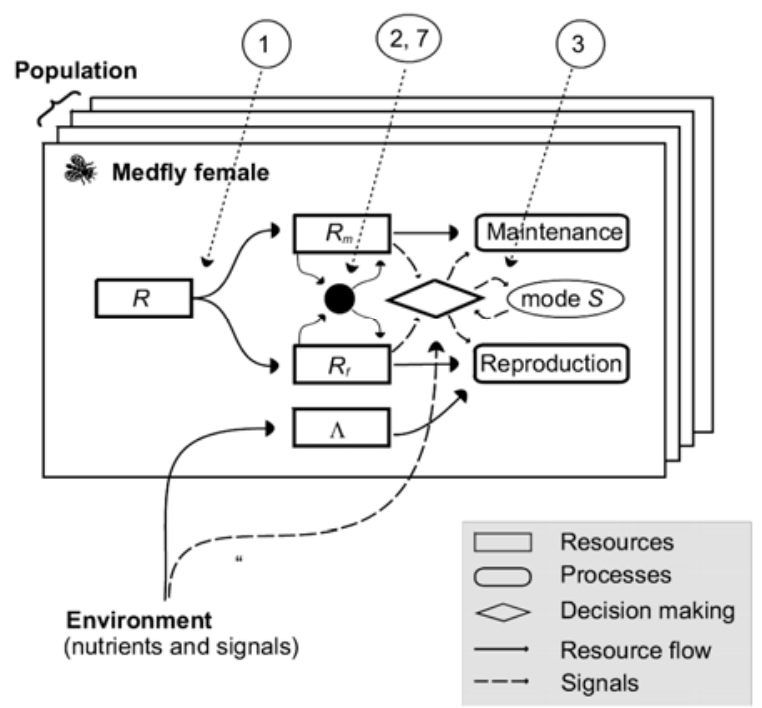

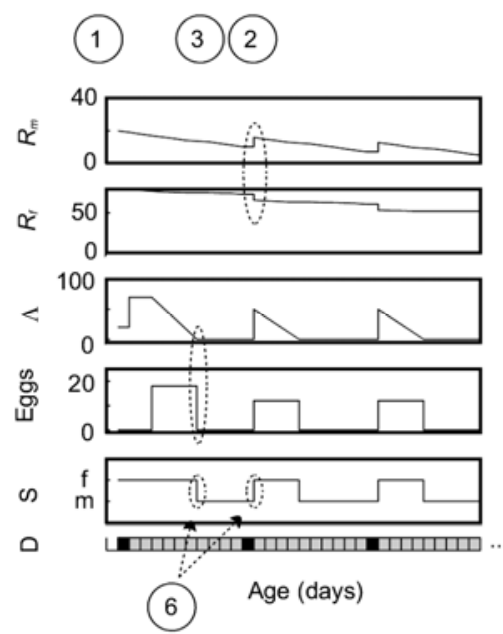

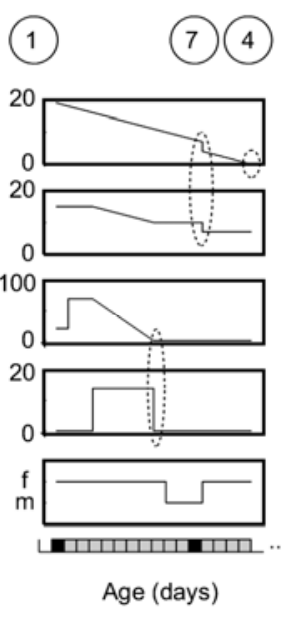

Fig. 3. Left panel: metabolic mechanisms and related events described by the model. Right panel: example of resource reallocations occurring on diet consisting of 1 day of complete adult diet and 10 days of sugar-only diet (1:10): a) a long-lived, b) a short-lived fly. Circles denote type of event (see Table 2); only the first event of any kind is shown. Bottom horizontal plot is the time line: days on sugar are gray, on complete diet are black. Ellipses denote resource related events (reallocation, drop, and exhaustion). The initial value of dietary resource (inherited from larvae) $\Lambda_{0}>0$. The complete diet provides $\lambda=50 \mathrm{U}$ (units) of essential dietary resource $\Lambda$ daily. During reproduction it is spent with the rate $\alpha_{\lambda}(1 \mathrm{U} / \mathrm{egg})$. The reproduction resource $R_{f}$ is also spent with the rate $\alpha_{f}(0.05 \mathrm{MU} / \mathrm{egg})$. Maintenance resource $R_{m}$ decreases with the rate $\alpha_{m}$ ( $1 \mathrm{MU} /$ day). When the diet changes, a mode changes as well, and the resource reallocation occurs with probability $p$. The depletion of $\Lambda$, or $R_{f}$ stops the egg laying. When $R_{m}$ is depleted the fly dies. b) Attempt to switch the mode at low level of available metabolic resource results in overload of weak energy supply system resulting in its' drop (7), and fly's death (4).

\section{The population model}

We assume that the phenotypic differences in lifespan and fertility observed in the fly population result from the difference in level of initial metabolic resource and distribution and that the distribution of the level of metabolic resource $R_{0}$ in the population of newly born flies can be described by $\Gamma$-distribution. This distribution was selected because the random values are nonnegative and depending on parameters it can approximate exponential, uniform and normal distributions. $\Gamma$-distribution is often used for modeling the population heterogeneity. Assume that the initial level of $\Lambda_{0}$ also is $\Gamma$-distributed. Initial state $S$ is assumed to be random and take both 
values with equal probability. Values of the population model parameters are given in Table 3 .

\section{Model identification}

The model was identified using the data of experiments $\mathbf{p}, \mathbf{p 1 : 1}$ (1-1 cycle), p1:3 (1-3 cycle), and s. For each diet the average lifespan and fertility was evaluated in groups of all flies, shortand long-lived flies for experimental and model cohorts. The squared distances between the points of averages in model and data was summed across all groups and all diets and used as a measure of model fit (see Fig. 1$)^{2}$. Denote the individual lifespan and fertility $X$ and $F$ correspondingly. $\bar{X}$ and $\bar{F}$ will denote group-average values.

Different model parameters affect different characteristics of the solution which allows to identify them sequentially some after the others.

Parameter $\Lambda_{0}$ was estimated from reproduction in group $s$ since the initial resource level $\Lambda_{0}$ has a strong effect on reproduction of this group.

Parameters $\alpha_{f}$ and $c$ were estimated simultaneously. They control the averages in groups on diet $p$ : as $c$ increases more resource are allocated to reproduction; the decrease of "egg cost" $\alpha_{f}$ increases the number of eggs laid for the same resource expenditure. Both changes result in an increase of reproduction. Parameter $\alpha_{f}$ also controls the slope of regression line for the points of averages in groups L.

Initial value of $R_{0}$ affects the values of $\bar{X}$ and $\bar{F}$ in all samples. Increase of variance of $R_{0}$ increases the variance of $X$ and $F$ which results in decrease of values in group of short-lived, no change in all and increase in group of long-lived (see Fig. 1, circles).

The share of ration $p$ affects the usage of metabolic resource, the balance between lifespan and fertility, and variance of these values, this can be best noted in experimental groups L.

Three parameters $\lambda_{p}$, the amount of dietary resource assimilated daily on diet $p, \Lambda_{t}$, the maximum reserve of dietary resource, and $f_{\max }$, the maximum number of eggs laid daily (production of each egg requires one unit of dietary resource) have the following effect. Increasing $\lambda_{p}$ increases reproduction in all cohorts receiving $p$ if the reproductive resource allows. But when the ration $p$ if frequent, $f_{\max }$, the maximum number of eggs laid daily becomes limiting. This results in a saturation of daily reproduction as the food quality increases.

When the ration $p$ is rare the maximum reserve of dietary resource $\Lambda_{t}$ becomes limiting. Parameter $f_{\max }$ controls how the metabolic resource is spent on egg laying. The model does not describe the daily dynamics of reproduction, its results are correct only on time-average. In the reproductive mode the flies were able to lay as many eggs daily as their metabolic $R_{f}$ and dietary resource $\Lambda$ allowed but not more than $f_{\max }$ eggs per day.

We assume that half of the model flies in cohort $\mathbf{p}$ begin their lives in maintenance mode $\mathbf{m}$ and experience only one mode change $\mathbf{m} \rightarrow \mathbf{f}$ hence one resource reallocation after eclosion. The second half of the flies in this cohort starts their lives in fertility mode $\mathbf{f}$ and experiences no switches. Similarly a half of the model cohort $\mathbf{s}$ starts life in the maintenance mode $\mathbf{m}$ and does not switch or reallocate resources. The second half begins life in reproductive mode $\mathbf{f}$ and switches once reallocating resource according to $c$.

The obtained parameter estimates were refined in a way that the distributions of lifespan in experimental groups $\mathbf{p}, \mathbf{s}$ and groups with cycle diets were closest to the modeled distributions in sense of likelihood.

Final values of parameters and variables of the life history model are given in Table 3 . A cohort

${ }^{2}$ The fertility had a weight 0.1 when calculating the distance to give them weight comparable to that of lifespan. 
of 10000 flies was modeled for each diet.

\section{Conclusion}

The construction of the model pursued two main objectives. The first was to digest the large body of knowledge about the physiology and biology of the fly, metabolic mechanisms related to maintenance and reproduction and to select the most important ones related to the experiment. The second aim was to build a simplest quantitative mechanical model of a single individual and of a population which would describe the processes of maintenance and reproduction support in Medfly females and capture the observed effects on individual and population levels. The model describes the noticed population-level effect (longevity-fertility trade-off) rather well (see Fig. 1.). The survival is different cohorts is also described acceptably considering the simplicity of the model. The individual dynamics of daily reproduction is described acceptably as well. Still the model is unable to capture the huge population heterogeneity in terms of fertility. But as with all such models there is always a place for an unexplained heterogeneity that occurs in nature but can not be adequately explained by a mechanistic model taking into consideration only certain features of the process. The further development of this work is to construct a continuous-time model which would describe longevity and fertility responses of a Medfly female to variations in food quantity and composition and would be based on description of metabolic mechanisms and limitations underlying these two fundamental traits of the organism. This work is continued in (Karkach, Romanyukha, in Press).

\section{Acknowledgements}

This work was supported by the Max Planck Institute for Demographic Research, Rostock, Germany.

\section{References}

Carey, J.R., Liedo, P., Muller, H.-G, Wang, J.-L., Vaupel, J.W., 1998. Dual Modes of Aging in Mediterranean Fruit Fly Females. Science 281, 996-998.

Carey, J.R., Liedo, P., Harshman, L., Liu, X., Muller, H.-G., Partridge, L., Wang, J.-L., 2002. Food pulses increase longevity and induce cyclical egg production in Mediterranean fruit flies. Functional Ecology 16, 313-325.

Harshman, L.G., Zera, A.J., 2007. The cost of reproduction: the devil in the details. Trends in Ecology \& Evolution 22: 2, 80-86.

Romanyukha, A.A., Carey, J.R., Karkach, A.S., Yashin, A.I., 2004. The impact of diet switching on resource allocation to reproduction and longevity in Mediterranean fruitflies. Proc. R. Soc. Lond. B. 271, 1319-1324.

Karkach A.S., Romanyukha A.A. The model of metabolic maintenance and its application to pulsed diet experiments on Medflies. Max Planck Institute for Demographic Research, working paper 2009 (in Press).

Romanyukha, A.A., Karkach, A.S. Yashin A.I. Adaptive trade-off in C. capitata is a characteristic feature of the long-lived subpopulation. Max Planck Institute for Demographic Research, working paper 2009 (in Press).

Simmons, F.H., Bradley, T.J., 1997. An analysis of resource allocation in response to dietary yeast in Drosophila melanogaster. Journal of Insect Physiology 43, 779-788. 